\title{
UNA ALTERNATIVA DE LA IZQUIERDA FENOMENOLÓGICA A LA TEORÍA DE LA INTERPRETACIÓN JURÍDICA DE HART / KELSEN*
}

\author{
Duncan KENNEDY
}

\section{Resumen:}

En este artículo el autor presenta una alternativa a las teorías de la interpretación jurídica de H. L. A. Hart y de Hans Kelsen. En ese sentido, intenta, primero, recapitular las principales caracteristicas de las mismas; segundo, resumir la alternativa de la izquierda fenomenológica al positivismo jurídico; y, tercero, responder a algunas de las lecturas equivocadas de dicha alternativa.

\section{Abstract:}

In this article the author presents an alternative to the theories of legal interpretation of H. L. A. Hart and Hans Kelsen. In that sense, he aims, first, to recapitulate the main characteristics of such theories; second, to resume the left phenomenological alternative to legal positivism; and, third, to respond to some misreadings of that alternative.

* La primera versión de este artículo fue preparada para el Congreso sobre Problemas Contemporáneos de la Filosofia del Derecho organizado por la UNAM, en la ciudad de México, bajo el título "A Left Phenomenological Critique of the Hart/Kelsen Theory of Legal Interpretation" y publicado bajo ese título en un libro que recogió las ponencias del Congreso, Cáceres, Enrique et al. (eds.), Problemas contemporáneos de la filosofía del derecho, México, UNAM, Instituto de Investigaciones Jurídicas, 2005, pp. 371 y ss. Esa versión fue traducida al holandés y publicada como "Een linkse fenomenologische kritiek op de rechtsvindingstheorie van Hart en Kelsen", Nederlands tijdschrift voor Rechtsfilosofie \& Rechtstheorie, vol. 3, 2004, pp. 242 y ss. Ésta es una versión sustancialmente revisada. Gracias a Imer Flores y Brian Bix por sus comentarios útiles. Los errores son nada más míos. Traducción de Imer B. Flores y Roberto Vidal Sánchez. 
Este artículo contiene tres partes. La primera presenta un resumen de algunos elementos comunes a las teorías de la interpretación jurídica de H. L. A. Hart y Hans Kelsen. La segunda resume la alternativa de la izquierda fenomenológica al positivismo jurídico, tal y como fue desarrollada por una tendencia dentro de los estudios críticos jurídicos (CLS). La tercera intenta clarificar dicha alternativa mediante la respuesta a una de las muchas lecturas equivocadas de la posición de CLS que son comunes en las corrientes positivistas y pospositivistas dominantes en la filosofia jurídica académica estadounidense.

Esta parte se refiere a la idea común en los breves escritos canónicos de Hart y Kelsen sobre interpretación jurídica. ${ }^{1}$ Imaginamos la norma que ha de ser interpretada como un área o espacio con dos partes. En Hart hay un "centro/núcleo de certeza" y una "penumbra de duda", también conocida como un "halo de vaguedad" y una "área de textura abierta". ${ }^{2}$ En Kelsen hay un "marco, el cual comprende varias posibilidades de aplicación". ${ }^{3}$ Dentro del centro/núcleo hartiano la interpretación es "determinada". En la penumbra es una cuestión de discreción, de ponderación de consideraciones conflictivas, de legislación judicial, o de creación del derecho. Afuera del marco kelseniano, la norma es determinada, pero dentro del marco hay interpretaciones alternativas de entre las cuales el juzgador debe elegir, al ejercer su discreción, crear derecho o ponderar intereses, y en consecuencia la interpretación no es determinada.

1 Hart, H. L. A., The Concept of Law, Oxford, Oxford University Press, 1961 (de aquí en adelante: Hart) [hay versión en español: El concepto de derecho, trad. Genaro R. Carrió, Buenos Aires, Abeledo-Perrot, 1963 (N. T.); y, Kelsen, Hans, Introduction to the Problems of Legal Theory, trad. B. Paulson \& S. Paulson, Oxford, Oxford University Press, 1992 (1934) (de aquí en adelante: Kelsen).

2 Hart, op. cit., nota 1, por ejemplo, pp. 119-20, 123-26, 128, 131, 135, 143, 150.

3 Kelsen, op. cit., nota 1, pp. 77-81. 
Para Kelsen y Hart la determinación de una norma dada, vista en su conjunto, es un asunto de grado. Para Kelsen, las normas constitucionales que definen el ejercicio apropiado del Poder Legislativo son relativamente indeterminadas respecto a qué leyes la legislatura debería adoptar, mientras que las leyes son relativamente más determinadas respecto al contenido de las decisiones judiciales que pretenden ser aplicables. De la misma manera, para Hart las normas pueden tener penumbras más grandes o pequeñas, aunque algunos estándares (p. ej. "tarifa o precio justo") tienen significados en el centro/núcleo.

Me parece que para los positivistas debería ser importante que tanto para Hart como para Kelsen la existencia de un área de indeterminación para las normas es inevitable, y que ninguno de los dos proporciona una explicación clara del por qué esto debería ser verdadero. Sin embargo, en cada caso, el uso del imaginario espacial es un obstáculo mayor para el entendimiento de qué quieren decir. ${ }^{4}$ Pero nada en la discusión me parece girar en torno a cómo interpretarlos respecto a esta cuestión.

Es de la misma manera sorprendente que ambos autores parecen usar las frases determinada/o en una forma confusa. Algunas veces significa solamente que se puede predecir con gran certeza lo que el intérprete hará con el problema ante sí. ${ }^{5}$ En otras ocasiones significa que la operación es "cognitiva", en el sentido de que la entendemos como un juicio acerca de un significado, entendido como algo que es independiente del observador, y respecto al cual creemos que existe una "verdad de la cuestión", incluso si los intérpretes

4 Véase las discusiones útiles en Gianformaggio, Letizia y Paulson, Stanley (eds.), Cognition and Interpretation of Law, Turin, G. Giappichelli, 1995.

5 Hart, op. cit., nota 1, por ejemplo, p. 123: "Los casos claros, en que los términos generales parecen no necesitar interpretación y el reconocimiento de los ejemplos puede ser "automático", son únicamente los casos familiares que se repiten en forma constante en contextos semejantes, respecto de los cuales existe acuerdo general sobre la aplicabilidad de los términos clasificatorios". Para una discusión útil, véase Bix, Brian, Law, Language and Legal Determinacy, Nueva York, Oxford University Press, 1993. 
están probablemente en desacuerdo acerca de cuál es la verdad. ${ }^{6}$ Otra vez, esto no será un problema en el desarrollo.

Lo que será importante en el análisis siguiente es que, para los casos en el área de certeza, hablan como si la cognición de un significado correcto para el centro/núcleo o marco, o la elección altamente predecible de una interpretación, fuera automática y fácil, al suponer la buena fe. Kelsen parece completamente auto-inconsciente al asumir que la cuestión está o no está "dentro del marco". Como un asunto de "cognición". 7 Hart está más matizado. Para él, "en los casos concretos particulares pueden surgir dudas" como los casos de ambigüedad "que la naturaleza o la inventiva humana continuamente presentan". ${ }^{8}$ Pero cualquier caso dado meramente está, no obstante, siempre localizado en un espacio metafórico o en otro.

Quizás por esta razón, cada autor es algunas veces caracterizado como un "formalista". Cuando afirman que, en una situación particular, hay una sola respuesta correcta a la cuestión interpretativa, se leen como literalistas, a pesar de lo insistentes que puedan ser acerca de la naturaleza discrecional de las cuestiones localizadas en la penumbra o en el marco. Es claro, no obstante, que cada autor se considera a sí mismo como un enemigo determinado del modo de razonamiento jurídico que fue llamado formalismo en su tiempo, a saber la jurisprudencia conceptual.

La jurisprudencia conceptual acepta que habrá situaciones en las cuales hay más de una norma válida (sección del código o precedente obligatorio) que, considerado por separado, es discutiblemente aplicable a los hechos, y que las normas diferentes darán resultados distintos al caso. Los

6 Hart habla de "ejemplos claros no discutibles de casos que los satisfacen o que no los satisfacen [los estándares generales]". La parte del "acuerdo general" ha desaparecido en esta cita. Hart, p. 128. Cuando Kelsen discute la utilización de consideraciones extra-jurídicas en el momento de la creación de derecho dentro del marco, habla de "espacio para una actividad cognitiva más allá de descubrir el marco dentro del cual el acto de aplicación se encuentra confinado". Kelsen, op. cit., nota 1, p. 83 .

7 Kelsen, véase nota 6 supra.

8 Hart, op. cit., nota 1, p. 123. 
juristas conceptuales (y sus críticos, por ejemplo, Gény en el Méthode d'interpretation et sources en droit prive positif) ${ }^{9}$ han tendido también a creer que hay situaciones que son "nuevas" en el sentido específico de que ninguna norma jurídica válida fue pensada específicamente para determinarlas de una manera u otra.

$\mathrm{Su}$ método requiere que el juzgador encare tanto conflictos como lagunas del modo siguiente: él va a presuponer la coherencia "del sistema" como un todo, y entonces preguntar cuál de las normas en conflicto, o qué norma nueva, aplicable al caso, "encuadra" mejor con las normas estrechamente relacionadas. Si esto no está claro, se mueve a las normas más abstractas, explícitas o implícitas en "el sistema", de las cuales las normas particulares se entiende que derivan (Savigny). ${ }^{10}$ Otra vez, escogerá una norma o diseñará una nueva norma, pero sin ejercer su discreción, ponderación, o "creación del derecho" en el sentido de legislación.

Desde el punto de vista de H/K, la operación de "construcción", por medio de la cual el jurista conceptual enfrenta el conflicto o la laguna, es discrecional y "legislativa". Consideran que la jurisprudencia conceptual sobreestima la determinación del sistema jurídico, ya sea que tratemos con normas aisladas o con "el sistema" tomado como un todo. Una virtud mayor del positivismo, como lo entienden, es el reconocer e incluso subrayar la creación judicial del derecho que el conceptualismo ofusca.

Junto con el literalismo y la jurisprudencia conceptual, el tercer método de interpretación de las normas jurídicas, que es común en el dominio jurídico occidental, es el análi-

9 Gény, François, Méthode d'interpretation et sources en droit prive positif: essai critique, 2a. ed., París, Librairie generale de droit et de jurisprudence, 1919 [hay versión en español: Método de interpretación y fuentes en derecho privado positivo, Madrid, Reus, 1925 (N. T.)].

10 Savigny, Friedrich Karl von, System of the Modern Roman Law, trad. W. Holloway, Madras, J. Higginbotham, 1867 [hay versión en español: Sistema de derecho romano actual, trad. Jacinto Mesia \& Manuel Poley, Madrid, Góngora, s.a., VI vols.; y hay reimpresión: Granada, Comares, 2005 (N. T.)]. 
sis de políticas, o el método de ponderar o de proporcionalidad. Aquí, el intérprete considera que él mismo tiene una elección entre distintas normas o formulaciones de la norma, una elección que se resuelve al apelar a las consideraciones conflictivas que él entiende subyacen al sistema normativo como un todo.

Hay muchas variantes del método del análisis de políticas. Lo que se pondera podrian ser derechos, poderes, principios, o metas instrumentales, supuestamente del interés común, junto con intereses administrativos (por ejemplo, seguridad $v$ s. flexibilidad equitativa), y los intereses de la arquitectura del sistema (por ejemplo, subsidiariedad, o la separación de poderes). O todos los anteriores. ${ }^{11}$

Hart se refiere con aprobación a la ponderación de esta clase como el método apropiado para la penumbra o el área de textura abierta. ${ }^{12}$ Kelsen meramente apunta que no es una solución a un problema de discreción porque "no proporciona un estándar objetivo de acuerdo con el cual los intereses en competencia puedan ser comparados unos con otros". 13

Para CLS, el punto importante es que el anti-formalismo de $\mathrm{H} / \mathrm{K}$ presupone el esquema de acuerdo al cual cada caso está localizado ya sea en un área de determinación o en una penumbra o marco. Para nuestros propósitos, lo que cuenta no es que el análisis de politicas es requerido frecuentemente y apropiado, sino que no proporcionan una explicación de cómo la situación es enmarcada como una localizada en la

11 Véase, en general, Kennedy, Duncan, "From the Will Theory to the Principle of Private Autonomy: Lon Fuller's Consideration and Form", Columbia Law Review, vol. 100, 2000, pp. 94 y ss.; Kennedy, Duncan, "The Disenchantment of Logically Formal Legal Rationality, or Max Weber's Sociology in the Genealogy of the Contemporary Mode of Western Legal Thought", Hastings Law Journal, vol . 55, 2004, pp. 1031 y ss.; Kennedy, Duncan y Belleau, Marie-Claire, "La place de René Demogue dans la génélogie de la pensée juridique”, Revue Interdisciplinaire D'études Juridiques, vol. 56, 2006, pp. 163 y ss.

12 Hart, op. cit., nota 1, por ejemplo, pp. 126-30.

13 Kelsen, op. cit., nota 1, p. 82. 
penumbra o el marco, de forma tal que no hay una respuesta determinada disponible.

En otras palabras, antes de que el análisis de políticas comience, cualquiera que sea su contenido, el intérprete enmarca explícita o implícitamente la situación como una en la cual hay un conflicto o una laguna que lo exime del deber elemental de aplicar una norma clara cuando los hechos encuadran claramente dentro de sus definiciones. $\mathrm{H} / \mathrm{K}$ se parecen a los juristas conceptuales y a los inventores de la ponderación de intereses en que no han teorizado este encuadre inicial.

II.

Esta parte, primero, ofrece una explicación del proceso por el cual los intérpretes constituyen situaciones jurídicas en cualquiera de las dos formas siguientes: ya sea como unas en las cuales todo lo que se requiere es la aplicación de una norma, o como unas en las cuales, porque estamos en la penumbra o dentro del marco kelseniano, o hay un conflicto o una laguna, algo más que la mera aplicación de una norma es requerida (el "algo más" es una elección de entre las interpretaciones elegibles basadas en la discreción legislativa, en el análisis de coherencia, en el análisis teleológico, en el análisis de políticas o en lo que sea). Segundo, ofrece una explicación del rol de la "ideología" en el proceso de encuadrar y después decidir asuntos que implican "intereses" significativos. Tercero, aplica esta concepción a la cuestión de cómo entender el rol de la ideología en el cambio jurídico y la estabilidad jurídica. ${ }^{14}$

14 Esta sección es en gran parte un resumen del enfoque propuesto en Kennedy, Duncan, "Freedom and Constraint in Adjudication: A Critical Phenomenology", Journal of Legal Education, vol. 36, 1986, pp. 518 y ss. [hay versión en español: Libertad y restricción en la decisión judicial, trad. Diego Eduardo López Medina y Juan Manuel Pombo, Santa Fe de Bogotá, Siglo del Hombre Editores, 1999 (N. T.)]; y en Kennedy, Duncan, A Critique of Adjudication [fin de siecle], Cambridge, Massachusetts, Harvard University Press, 1997, Partes $1 \& 3$. 
A.

En el marco teórico de Hart/Kelsen, compartido por la jurisprudencia conceptual y por el análisis de políticas, no hay espacio para la actividad que colocaria en medio de una fenomenología de centros/núcleos, marcos, lagunas y conflictos, una fenomenología que pueda explicar la determinación e indeterminación. Esta es la actividad de "trabajo" jurídico entendida como la transformación de una aprehensión inicial (Husserl) ${ }^{15}$ de lo que los materiales jurídicos que componen al sistema requieren, por un actor que persigue una meta o una visión de lo que deberian requerir (esta concepción del trabajo está inspirada por los Manuscritos económicos y filosóficos de 1844-45 de Marx). ${ }^{16}$

El trabajo jurídico, como uso el término, ya sea que se dirija a centros/núcleos o a marcos, a penumbras o conflictos o lagunas, es entendido "estratégicamente". El trabajador intenta transformar una aprehensión inicial de lo que el sistema de normas requiere, dados los hechos, asi que la aprehensión nueva del sistema, en tanto aplique al caso, corresponderá a las preferencias extra-jurídicas del trabajador interpretativo.

El trabajo jurídico ocurre después de la aprehensión inicial de hechos y norma, y después de la "aplicación de la regla auto-inconsciente". El intérprete "comprende" (un proceso gestáltico, como en la Psicología Gestáltica de Kohler) ${ }^{17}$ la situación en su conjunto como una en la cual una norma gobierna y la pregunta es si los hechos particulares dentro de la situación provocan su aplicación para producir

15 Husserl, Edmund, "The Natural Attitude and its Exclusion" (1913), en Welton, Donn (ed.), The Essential Husserl: Basic Writings in Transcendental Phenomenology, Bloomington, Indiana University Press, 1999, p. 60.

16 Marx, Karl, "Economic and Philosophical Manuscripts" (1844) en Easton, L. y Guddat, K. (eds.), Writings of the Young Marx on Philosophy and Society, trad. L. Easton \& K. Guddat, Nueva York, Doubleday, 1967 [hay versión en español: $M a^{-}$ nuscritos económicos-filosóficos de 1844, trad. Wenceslao Roces, México, Grijalvo, 1968 (N. T.)].

17 Kohler, Wolfgang, Gestalt Psychology: An Introduction to New Concepts in Modern Psychology, Nueva York, New American Library, 1947. 
una sanción. Alguien ha muerto, y la corte se pregunta, primero, si el acusado mató a una persona, y, segundo, si dicho asesinato fue un homicidio, pero eso "depende de los hechos". Con frecuencia, una vez que los hechos son encontrados, nadie advertirá la posibilidad del trabajo jurídico dirigido a la interpretación de la norma que define y castiga el homicidio. Los hechos se entenderán que establecen la culpabilidad o la inocencia "de su propio modo", como la "norma se aplica a sí misma" aparentemente sin la agencia del intérprete.

Es común que los hechos cobren existencia jurídica mediante el trabajo de los investigadores, así que los hechos presentados dependen de las estrategias de trabajo y de los niveles de esfuerzo de los acusadores y de las partes. También es común que los abogados y el juez, y, en un nivel más abstracto, el jurista, trabajen a veces para transformar la aprehensión inicial de cuál norma es aplicable y qué requiere. Esto es "conducta estratégica en la interpretación".

Éstos son tres tipos de comportamiento estratégico en la interpretación:

Primero, tratar de encontrar los argumentos jurídicos que producirán el efecto de necesidad jurídica para un resultado - esto es, para una regla-aplicable-a-los-hechos-dadosdiferente del resultado que apareció inicialmente requerido como auto-evidente, como, por ejemplo, hacer parecer que hay por necesidad una excepción a la regla que cubre el caso en apariencia, o que el "significado verdadero" de la regla es diferente del que parecia ser en un principio.

Segundo, tratar de hacer pasar lo que parecía ser una decisión judicial discrecional auto-evidente (una en la penumbra o dentro del marco) como una en la que hay, a final de cuentas y contra-intuitivamente, un resultado particular -una regla-como-es-aplicada-a-los-hechos- que es requerido por los materiales (i. e. el caso cae dentro del centro/núcleo; o no hay alternativas dentro del marco).

Tercero, tratar de desplazar un resultado jurídico requerido inicialmente como auto-evidente con la percepción de la 
situación como una en la cual el juzgador está obligado a escoger de entre alternativas jurídicamente permisibles (i. $e$. mover una interpretación del centro/núcleo a la periferia o dentro del marco que permite la discreción judicial).

En todos estos casos, el intérprete trabaja para crear o deshacer la determinación, en lugar de simplemente registrarla o experimentarla como algo dado por la situación.

El trabajo presupone un medio, algo que el trabajador "confecciona". En este caso, el medio es ese cuerpo de materiales jurídicos que se consideran relevantes para establecer el significado de la norma como aplicable a los hechos. Esto ciertamente incluirá el diccionario con sus definiciones, el diccionario jurídico con sus voces bastante diferentes, el comentario doctrinal, y el cuerpo completo de normas jurídicas válidas, quizás derechos y principios más abstractos, quizás debates legislativos, quizás casos y precedentes. Desde nuestro punto de vista, la pregunta no es qué cuenta, oficialmente, como "fuentes", sino qué elementos se buscan y se despliegan de hecho en el trabajo de abogacía o justificación.

El trabajo del trabajador usa los materiales jurídicos para convencer a una audiencia de alguna clase (y a él mismo también) que una aprehensión inicial (la suya o la de otro) de la determinación o la indeterminación estaba equivocada. Sin embargo, no hay nada que garantice que dicha empresa triunfará. El trabajo no es cognición de la ley obligatoria ni discreción para divisar el derecho de acuerdo con sus "preferencias legislativas". Está entre ambos. Los materiales jurídicos restringen el trabajo jurídico pero en la forma en la cual un medio constriñe a cualquier trabajador. Solamente restringe en contra de un esfuerzo por hacer que los materiales signifiquen cualquier cosa.

Decir que la interpretación de la regla era determinada quiere decir solamente que al final del proceso de trabajo el intérprete fue incapaz de alcanzar la re-interpretación estratégica deseada a partir del significado inicialmente autoevidente manifiesto de la norma como aplicable a los he- 
chos. En otras palabras, los estudios críticos jurídicos, como los entiendo, aceptan por completo la idea positivista de que el derecho es en algunas ocasiones determinado y otras veces indeterminado. CLS rechaza tanto la idea de la indeterminación global como la idea de que siempre hay una interpretación correcta, sin importar que tan oscura o difícil sea de alcanzar. Pero rechaza además la idea de que la determinación y la indeterminación sean "calidades" o "atributos" inherentes en la norma, independientemente del trabajo del intérprete.

El éxito estratégico en contra de la determinación (o indeterminación), inicialmente auto-evidente es una función de tiempo, estrategia, habilidad, y de los atributos "intrinsecos", "esenciales", "objetivos" o "reales" de la regla que se trata de cambiar, como aparecen en el contexto de los hechos presentados.

La pregunta "ontológica" es si es apropiado considerar la determinación de la regla como aplicada a los hechos, lo cual significa su calidad obligatoria o "válida" como insuperable al final del periodo que se tuvo para trabajar con ella, como su atributo propio, o como algo inherente a ella. La alternativa es que la calidad determinada o indeterminada de la regla no se puede entender de otro modo que como un "efecto", "el efecto de la necesidad" o el "efecto de la determinación", producido contingentemente por la interacción del tiempo, estrategia y habilidad del intérprete con un ininteligible "existir en sí" o la naturaleza "esencial" de la regla en un contexto fáctico dado.

E1 trabajador jurídico realiza la reducción fenomenológica clásica o "poner entre corchetes" [epoché] (Husserl)18 la cuestión de si la resistencia de la regla a la reinterpretación es un resultado de lo que "en realidad" es o meramente un efecto del tiempo, estrategia y habilidad. El trabajador procede a tratar de cambiar las cosas, sin un pre-compromiso de una forma u otra con una ontología de la norma. Para el intérprete estratégico nada surge de decidir sobre la esen-

18 Husserl, op. cit., nota 15, p. 65. 
cia. La posición de la izquierda fenomenológica dentro de CLS adopta también esta actitud.

B.

Los intereses determinan cuánto trabajo hay que realizar. La distinción de Max Weber entre intereses materiales e ideales es aplicable. ${ }^{19}$ Los litigantes pueden estar motivados materialmente, y el juez también, pero los jueces (y los juristas) están con frecuencia obviamente conscientes sólo de intereses ideales. Ellos escogen una estrategia de trabajo porque entienden que su empresa ha de tener que ver con la "justicia", entendida como no-idéntica con la aplicación de la ley. Ellos también entienden que su deber es alcanzar la justicia "conforme" al derecho. Pero este deber puede ser operativo solamente después que la ley es establecida. La definición convencional del papel judicial (o jurístico) no dice nada acerca del trabajo jurídico, porque el modelo tradicional (positivista) reconoce solamente cognición y discreción, y no deja lugar para trabajo.

Aquéllos que comprenden la interpretación como cognitiva o discrecional, probablemente consideran al trabajo diseñado para lograr un cambio particular en el significado auto-evidente de una norma, en una dirección que es determinada estratégicamente, esto es, extra-jurídicamente, como ilegítimo. Considero que el argumento sobre la ilegitimidad es incorrecto.

Primero, la mayoría de las personas están de acuerdo en que se supone que los jueces trabajan en la interpretación, y tienen que decidir cómo orientar su trabajo. Ciertamente, la mayoria de los juristas considerarían como una violación a los deberes de la función judicial que el juez actúe simplemente sobre el significado de la norma que era inicial-

19 Gerth, H. H. y Wright Mills, C., "Introduction", From Max Weber: Essays in Sociology, Nueva York, Oxford University Press, 1946, pp. 61-65 [hay versión en español: "Introducción", Ensayos de sociología contemporánea, trad. Mireia Bofill, Barcelona, Martínez Roca, 1972 (N. T.)]. 
mente auto-evidente, una vez que se ha indicado que hay otra posibilidad. La razón de esto es que el juzgador conoce que ese trabajo puede cambiar la apariencia inicial. Él no puede tomarlo como meramente "verdadero" porque es autoevidente desde un inicio que es legal.

Ante la obligación de trabajar en una dirección u otra, los jueces (y los juristas) a menudo optan por orientar su trabajo hacia la meta de hacer que su intuición extra-jurídica o legislativa de la elección-de-la-justicia-en-regla sea la realidad de la decisión judicial; estos jueces son los "activistas", en el lenguaje de los estadounidenses. En la conciencia legal contemporánea, los jueces que trabajan de esta forma son acusados de hacer este trabajo "ideológicamente".

En el discurso jurídico contemporáneo, una ideología es un "proyecto de universalización" (Habermas), 20 lo cual significa la afirmación de una concepción controversial de la justicia, criticada por algunos como mera racionalización de intereses partidarios, pero defendida por sus adeptos por servir a los intereses de todos - asi como los intereses alegados por sus adversarios como meramente parciales (Mannheim)-.21

La persecución de una agenda jurística ideológica es abiertamente problemática para un juez o un jurista, porque aun si se reconoce sin titubear que los jueces están obligados por su función a trabajar para hacer corresponder el derecho positivo con la justicia, es una premisa de la teoría democrática liberal de la separación de poderes que esa ideología no es para la judicatura (ni para el jurista) sino para la legislatura electa democráticamente.

20 Habermas, Jurgen, The Theory of Communicative Action: Reason and the Rationalization of Society, trad. T. McCarthy, Boston, Beacon Press, 1984, vol. I, pp. 16-19 [hay versión en español: Teoría de la acción comunicativa: Racionalidad de la acción y racionalidad social, Madrid, Taurus, 1987 (N. T.)].

21 Mannheim, Karl, Ideology and Utopia: An Introduction to the Sociology of Knowledge, Nueva York, Harcourt Brace, 1936 [hay versión en español: Ideologia y utopía. Introducción a la sociología del conocimiento, trad. Salvador Echavarría, México, Fondo de Cultura Económica, 1941; y trad. Eloy Terrón, Madrid, Aguilar, 1958 (N. T.)]. 
Los jueces con frecuencia responden al dilema al afirmar que trabajan, y tratan de trabajar, no-ideológicamente - al poner entre corchetes sus preferencias legislativas al decidir en cuál dirección tratarán de mover los marcos o centros/núcleos-. Pero cuando hacen esto, tienen que confrontarse con el hecho de que su audiencia, y ellos mismos, entienden que los resultados diferentes responden, en muchos casos con intereses grandes, a ideologias distintas.

Dos posturas judiciales (y jurídicas) muy comunes, ante este dilema, son la "bipolaridad" y la "separación de las diferencias". En la primera, el juez establece, para sí mismo y para otros, que él es "neutral" a la ideología porque alternan imprevisiblemente entre las alternativas definidas por las ideologías en conflicto. En segundo lugar, el juez establece su neutralidad al ser un "centrista", al divisar una solución que dé algo a cada lado, pero no da a ningún lado todo lo que demanda. Éstas son soluciones de mala fe, en el sentido de Sartre en el Ser y la Nada, ${ }^{22}$ porque ellos evitan el conflicto mediante su negación (en el sentido de Freud $^{23}$ y Anna Freud ${ }^{24}$ ).

La posición del juez "activista", quien consciente o inconscientemente sigue sus propios compromisos ideológicos (en lugar de alegar su neutralidad porque es un comodín o un centrista), parece más éticamente plausible. El juez conoce qué trabajo puede hacer que la regla se acerque a su preferencia legislativa, pero puede no hacerlo. Supongamos que está comprometido a aplicar la regla si él no la puede desestabilizar al utilizar las técnicas judiciales aceptadas y convencionales —esto es mediante la investigación de los materiales jurídicos llegará a su reinterpretación de acuerdo con los cánones aceptados del razonamiento jurídico-.

22 Sartre, Jean-Paul, Being and Nothingness: An Essay on Phenomenological Ontology, trans. H. Barnes, Nueva York, Citadel, 1965 [hay versión en español: El ser y la nada, trad. Juan Valmar, Buenos Aires, Losada, 2005 (N. T.)].

23 Freud, Sigmund, Sexuality and the Psychology of Love, Nueva York, Collier Books, 1963.

24 Freud, Anna, The Ego and the Mechanisms of Defense, trad. C. Baines, Londres, International Universities Press, 1937. 
¿Entonces por qué no debería dirigir su trabajo, tiempo, estrategia y habilidad, a encontrar el argumento que hará corresponder la ley a su concepción de justicia? Parece que él simplemente actuaria de forma ilegítima si él falla al intentar hacerlo; en otras palabras si él fracasó al tratar de intentar retrabajar el derecho positivo para hacerlo corresponder a su idea de justicia. El papel judicial (y jurístico) requiere fidelidad al "derecho" en el sentido complejo que combina un elemento positivo y uno ideal. Esta posición, la cual legitima el trabajo jurístico que intenta inclinar el derecho en la dirección ideológica preferida por el juez (o el jurista), es, por supuesto, "anárquica" (o por lo menos "plural") desde el punto de vista "jacobino" que localiza la legitimidad jurídica únicamente en la voluntad del pueblo.

\section{C.}

Si se reconoce que los jueces pueden y trabajan para cambiar los centros/núcleos o los marcos (ya sea que se considere este trabajo como legítimo o no), entonces una noción básica de Hart/Kelsen es socavada. Esto es lo que Kelsen llama la "concepción dinámica", ${ }^{25}$ en la cual el movimiento de la creación de la norma va de lo abstracto a lo particular o concreto. En Hart, es la noción de que la adjudicación "colma/llena" la periferia, también expresado por MacCormick en esta cita:

La tesis de que aún las mejores leyes o lineas dibujadas dejan alguna penumbra de duda, y esto llama para un ejercicio de discreción en parte politica para resolver la duda, no es particularmente nueva, es la moneda común del positivismo jurídico moderno...

Un punto crucial, empero, es que uno no debe perder ni subestimar el significado del dibujar-líneas o de la determinatio como se discutió. El derecho realmente resuelve y real- 
mente puede resolver asuntos de la prioridad entre principios al fijar las reglas, e incluso cuando surgen los problemas de interpretación, éstos se enfocan en puntos más estrechamente definidos de interpretar las reglas que si el asunto fuera uno de puro principio. Fijar las reglas puede hacerse tanto por la legislación como por el precedente; más comúnmente, en un sistema moderno, por los dos en combinación. Es uno de los obsequios del derecho a la civilización que puede, sujetar las preguntas prácticas a formas más estrechamente enfocadas de argumento que aquellas que están disponibles a la razón práctica sin restricción. ${ }^{26}$

Si el trabajo estratégicamente dirigido en la interpretación puede deshacer las aprehensiones iniciales de centros/núcleos o marcos, entonces esta afirmación es demasiado optimista acerca de los "legados del derecho a la civilización". En un desarrollo más extenso del tema, ${ }^{27}$ he propuesto que las "pequeñas" preguntas pueden tener intereses ideológicos muy grandes. Segundo, he sugerido, contrario a MacCormick, que los mismos argumentos de principio son recurrentes en cada nivel de abstracción, así que resolver asuntos "más abajo" en la pirámide implicará argumentos no menos polémicos que aquéllos situados en la cima. Este es el argumento acerca del fenómeno de "anidar", discutido en algún otro lugar. ${ }^{28}$

No obstante, para nuestros propósitos, aquí hay un punto bastante diferente: incluso después de que una interpretación es asentada, el trabajo puede desestabilizarla. Esto significa que el trabajo puede "infligir" o "cambiar" los centros/núcleos y los marcos. Hay ahora un "de abajo hacia

26 MacCormick, Neil, "Reconstruction after Deconstruction: A Response to CLS”, Oxford Journal of Legal Studies, vol. 10, 1990, pp. 553 y 554.

27 Kennedy, Duncan, A Critique of Adjudication [fin de siecle], cit., nota 14, pp. 172 у 173.

28 Kennedy, Duncan, "A Semiotics of Legal Argument", Syracuse Law Review, vol. 42, 1991, p. 75. Hay una version extendida en Collected Courses of the Academy of European Law, Amsterdam, Kluwer Academic Publishers, 1994, vol. 3, libro 2, pp. 309-365. 
arriba" dinámico contrapuesto hasta cierto punto $u$ otro con la dinámica "de arriba hacia abajo", de lo abstracto a lo concreto, de Hart y Kelsen. En lugar del enfoque progresivamente más estrecho para los asuntos controvertidos de MacCormick, el trabajador puede esperar dividir los centros/núcleos abiertos o disolverlos.

Así que el trabajo hace más que llenar el marco o la penumbra dinámicamente con elecciones de norma determinadas de manera estratégica. La ideología inflige el trabajo, el cual a su vez inflige en los marcos y centros/núcleos, los cuales proporcionan en cambio, en la perspectiva de la coherencia, medios para futuras desestabilizaciones de otros centros/núcleos y marcos.

Desde esta perspectiva, el conjunto de derecho válido, esto es el derecho que es considerado por los trabajadores jurídicos en su encuentro inicial con los materiales como centros/núcleos o marcos, es mejor entendido, primero, como un producto histórico del trabajo de abogados, juristas y jueces que han perseguido (en algunas ocasiones; consciente o inconscientemente) proyectos ideológicos conflictivos (los cuales pueden ser de centro, en el sentido de arriba), y, segundo, como siempre pero imprevisiblemente sujeto a desestabilización por futuras estrategias de trabajo futuro orientadas de manera ideológica.

\section{III.}

Para entender cómo la posición anterior representa una, posiblemente la posición dominante dentro de estudios críticos jurídicos desde alrededor de 1985, y, hoy, la única posición subsistente de CLS discutida de modo explícito, puede ser útil contrastarla con una típica lectura equivocada de CLS desde el interior de la corriente paradigmática de la filosofía jurídica anglo-americana, en este caso por mi amigo Brian Bix: 
[E]n particular, los teóricos de CLS han argumentado a favor de la indeterminación radical del derecho: el argumento de que los materiales jurídicos no determinan el resultado de casos particulares. Los teóricos de CLS aceptaron generalmente que los resultados de la mayoría de los casos eran predecibles; pero esto era, ellos afirmaron, no a causa de la determinación del derecho, sino porque los jueces tenían prejuicios conocidos y predecibles. Los materiales jurídicos, por sí mismos, se decía que eran indeterminados, porque el lenguaje era indeterminado, o porque las reglas jurídicas tendian a incluir principios contradictorios que permitieron a los jueces justificar cualquier resultado que ellos escogieron (Kelman 1987). Las criticas de CLS se han tenido generalmente por ser exageradas (Solum 1987); aunque bien puede haber casos para los cuales los materiales jurídicos no proporcionen un resultado claro, o al menos no un resultado en que todos podrian estar de acuerdo inmediatamente, esto no anula ni la facilidad de la inmensa mayoría de disputas posibles ni la posibilidad de respuestas correctas aún para los casos más difíciles. ${ }^{29}$

1. La tendencia de izquierda-fenomenológica de CLS (discutiblemente la tendencia dominante) argumentó que los materiales jurídicos determinan o no el resultado de los casos sólo en interacción con las estrategias argumentativas de juristas que persiguen objetivos con tiempo y recursos limitados. Los materiales son una parte de la determinación, pero sólo en combinación con la actividad interpretativa que no es cognitiva sino estratégica consciente o inconscientemente. No es y nunca fue la posición de esta tendencia dentro de CLS que los materiales "no determinan el resultado de casos particulares", sino que su influencia es mediada y que su determinación o indeterminación "intrínseca" o "esencial" es incognoscible.

29 Bix, Brian, "Law as an Autonomous Discipline", en Cane, Peter y Tushnet, Mark (eds.), The Oxford Handbook of Legal Studies, Nueva York, Oxford University Press, 2003, p. 983. La referencia a Kelman es Mark Kelman, A Guide to Critical Legal Studies, Cambridge, Massachusetts, Harvard University Press, 1987. La referencia a Solum es Solum, Lawrence, "On the Indeterminacy Crisis: Critiquing Critical Dogma”, University of Chicago Law Review, vol. 54, 1987, p. 462. 
Los materiales jurídicos son "indeterminados" sólo en el sentido de que algunas veces es posible desestabilizar las aprehensiones iniciales por medio del trabajo jurídico - "intrínseca" o "esencialmente" no son determinados ni indeterminados- Cierto, con frecuencia se aprehende inicialmente como determinando el resultado de un caso particular o, al contrario, como no determinando el resultado (porque el caso cae en la penumbra o dentro del marco). En el caso de la determinación aparente, se puede predecir los resultados cuando se adelanta que ningún trabajo se hará para desestabilizar la aprehensión inicial. Y a menudo será posible predecir que tal trabajo no se hará, porque los proyectos ideológicos existentes aplicados por la judicatura están de acuerdo con la aprehensión inicial, o de acuerdo con que el resultado no vale la pena del trabajo desestabilizador. Se podría anticipar lo opuesto si los actores con proyectos ideológicos radicales $\mathrm{u}$ otros proyectos periféricos trabajaran comúnmente como jueces o como juristas influyentes.

En un segundo momento, los materiales jurídicos son determinados en aquellos casos donde, después de que el trabajo jurídico ha llegado al grado de agotar el tiempo y los recursos disponibles, el intérprete se encuentra a sí mismo incapaz de desestabilizar la aprehensión inicial de que hay una norma aplicable y que esa norma decide el caso para una parte o la otra. Sobre esta base, se puede predecir los resultados cuando se adelanta que el trabajo hecho para desestabilizar la aprehensión inicial fracasará. En este caso, se hace una predicción acerca del resultado de la interacción entre los hechos como se presentaron, el trabajo interpretativo, y la "esencia" incognoscible de los materiales. Otra vez, las ideologías centristas compartidas por jueces y juristas en países capitalistas son un factor importante en esta clase de predicción.

Los escritores de CLS han trabajado desde el principio y continuamente para encontrar cómo las reglas que parecían susceptibles de resistir el esfuerzo más sostenido en la transformación por la vía de la interpretación, dadas las 
preferencias ideológicas de la izquierda moderada o de la derecha moderada compartidas virtualmente por todos los jueces en todos los países capitalistas, tienen impactos masivos e injustos en los grupos oprimidos. Esta es la contribución de CLS a la sociología del derecho y al análisis económico del derecho desde la izquierda.

2. La noción de que la indeterminación del lenguaje explicó la manera en que el derecho era indeterminado ha tenido alguna influencia en CLS, particularmente en los primeros escritos de Unger, ${ }^{30}$ y en escritores como Boyle, ${ }^{31}$ quien pretendia hablar de CLS como un todo. Desde el principio, una corriente más influyente argumentó que las reglas varían en "realización formal", o "aplicabilidad", así que la crítica lingüística simple es a menudo trivial, como son todos los demás argumentos de indeterminación "global". 32

La atribución de Bix a CLS de una noción de que "las reglas jurídicas contienen [o tienden a tener] principios conflictivos" es desconcertante. El argumento de CLS era, a la Dworkin, ${ }^{33}$ que los principios, las politicas y los derechos, son ciertamente visiones del mundo, son todos partes de las fuentes comúnmente desplegadas del derecho, pero, contra Dworkin, de que ellos están en conflicto inextricable, dentro de cada uno así como entre todos. Su presencia conflictual es reflejada en las más concretas "normas jurídicas válidas del sistema", las cuales CLS, al seguir el realismo jurídico, entiende como compromisos, siempre, complejos

30 Unger, Roberto Mangabeira, Knowledge and Politics, Nueva York, Free Press, 1975, p. 88 [hay versión en español: Conocimiento y política, trad. Leonardo Rodríguez Ozán, México, Fondo de Cultura Económica, 1985 (N. T.)].

31 Boyle, James, "The Politics of Reason: Critical Legal Theory and Local Social Thought”, University of Pennsylvania Law Review, vol. 133, 1985, pp. 710-11.

32 Kennedy, Duncan, "Legal Formality", Journal of Legal Studies, vol. 2, 1973, p. 351; y Kennedy, Duncan, "Form and Substance in Private Law Adjudication", Harvard Law Review, vol. 89, 1976, p. 1685.

33 Dworkin, Ronald, "The Model of Rules", Taking Rights Seriously, Cambridge, Massachusetts, Harvard University Press, 1977 [hay versión en español: "El modelo de las normas", Los derechos en serio, trad. Marta Guastavino, Barcelona, Ariel, 1984; y ¿Es el derecho un sistema de reglas?, trad. Javier Esquivel y Juan Rebolledo G., México, UNAM, Instituto de Investigaciones Filosóficas, 1977 (N. T.)]. 
de esos conflictos. ${ }^{34}$ Porque las reglas son compromisos, en lugar de un trabajo coherente desde un $u$ otro principio comprehensivo, ellos están mucho más abiertos a desestabilizaciones de varias clases de lo que reconocen los escritores del coherentismo.

3. Los "prejuicios" de los jueces son relevantes porque orientan el trabajo jurídico de jueces (y de otros juristas) al transformar las aprehensiones iniciales de lo que los materiales requieren en la dirección particular sugerida por los intereses materiales o ideales de los juristas (vagamente, la ideología del jurista). Que el jurista tendrá éxito en el trabajo de hacer que los materiales sean conforme a su estrategia extra-jurídica ideológica o material no es nunca cognoscible de antemano (aunque al igual que con algún acontecimiento futuro incierto, podemos hacer predicciones). Los juristas aceptan constantemente interpretaciones de acuerdo con las cuales el derecho positivo es contrario a su opinión de lo que debe ser.

Además, los "prejuicios" o la ideología no determinan las estrategias de trabajo de un jurista más determinantemente que cuando el sistema de normas jurídicas determina los resultados. Las ideologías son indeterminadas en la misma manera en la cual el orden jurídico lo es. Hay un círculo hermenéutico que funciona aquí, en el cual las indeterminaciones de cada nivel son resueltas al apelar a un nivel más profundo con sus propias indeterminaciones, y así sucesivamente, hasta regresar al punto de partida, en el cual las ideas jurídicas influyen en la ideología y viceversa. ${ }^{35}$

4. Las críticas de CLS se han considerado como sobre exageradas (o como muestras de incapacidad o locura mental) por parte de una corriente paradigmática que la ha entendido mal, más o menos a la manera de Brian Bix en la cita anterior. Dichas críticas son con frecuencia interpretadas mal, no como en los párrafos anteriores, sino al afirmar

34 Kennedy, Duncan, "From the Will Theory...", cit., nota 11; y "The Disenchantment...", cit., nota 11.

35 Kennedy, Duncan, A Critique of Adjudication..., cit., nota 14, pp. 187-91. 
"la determinación en la instancia final" en la base, o como una vulgar afirmación marxista de que los jueces son "el comité ejecutivo de la clase gobernante", y que proceden caso por caso para ampliar "los intereses del capital". Las malas lecturas derivan en parte de la más o menos completa ignorancia tanto de la fenomenología como de la teoría social crítica entre los teóricos jurídicos paradigmáticos estadounidenses, en parte por los recursos limitados que los filósofos jurídicos paradigmáticos dedican a corrientes marginales (Bix es excepcional en su familiaridad con los escritos de CLS), y en parte a la inversión normal de las corrientes paradigmáticas por reproducir la marginalidad de los marginados.

5. Todo el mundo sabe que "hay casos para los cuales los materiales jurídicos no dan un resultado claro". Y que hay casos en los cuales los materiales jurídicos no dan un resultado "en que todos estén inmediatamente de acuerdo". Pero es una cosa completamente diferente afirmar que hay una carga en las críticas del tipo de las de CLS para "negar" "la facilidad con que se resuelven la gran mayoría de disputas posibles". ${ }^{36}$ Esta afirmación cuantitativa tiene un lugar importante en el esquema positivista, al menos en el hartiano. Sirve para reafirmarnos que el reconocimiento de la discreción judicial en la penumbra no implica ninguna amenaza al valor liberal del Estado de derecho. Para Hart, el "escéptico ante las reglas" es bienvenido

...mientras no olvide que se le acepta en los lindes, y no nos ciegue frente al hecho de que lo que en gran medida posibilita estos notables desarrollos judiciales de las reglas más fundamentales, es el prestigio adquirido por los jueces a raíz de su actuación, incuestionablemente gobernada por reglas, en las vastas áreas centrales del derecho. ${ }^{37}$

36 Bix, Brian, op. cit., nota 29.

37 Hart, op. cit., nota 1, p. 150. Véase también pp. 149, 121-150, 132. 
El argumento de CLS es que la pregunta de qué proporción de las disputas actuales o imaginarias tienen resultados determinados, dados los materiales jurídicos, tiene que preguntarse al tomar en cuenta la posibilidad de que el trabajo jurídico desestabilice la aprehensión inicial de qué es lo que requieren los materiales. La determinación es una función no sólo de las palabras en que están formuladas las normas válidas y el contenido de otras fuentes, sino también de la interacción entre los recursos y estrategias de quienquiera que tenga el poder de hacer la interpretación jurídica, y la "cosificación" de los materiales y de los hechos como fueron presentados. Cuando se tome esto en consideración, las declaraciones acerca de "la inmensa mayoría de disputas" o "amplias áreas centrales del derecho" son simplemente un sin sentido. ${ }^{38}$

6. Que los resultados no sean determinados en algunos casos, según Bix, no "niega la... posibilidad de respuestas correctas aún para los casos más difíciles". El único significado inteligible de una "respuesta correcta" en un caso, difícil o fácil, dada la fenomenología expuesta, es que después de haber trabajado con el tiempo y los recursos disponibles y de acuerdo con una estrategia escogida, el intérprete no puede encontrar una alternativa a alguna aprehensión particular de a qué la regla aplica y qué requiere cuando aplica. En otras palabras, después de realizar la reducción fenomenológica, "la respuesta correcta" es la que es producida por un argumento que tiene "el efecto de necesidad". En cuanto a si hay una respuesta correcta en el sentido de una disponible para la cognición, CLS toma la posición de Kant respecto a "la cosa en sí".

38 Kennedy, Duncan, "Freedom and Constraint in Adjudication", cit., nota 14; y, A Critique of Adjudication..., cit., nota 14, p. 172. 